\title{
The Effect of Informal Central Bank Communication: Machine Learning Approach
}

\author{
Gytautas Karklius $^{1,2}$ (1)
}

Published online: 1 June 2018

(C) The Author(s) 2018

\section{JEL E40 $\cdot$ E50}

The management of agents' expectations is a key feature of monetary policy because the central bank only has direct control over very short-term interest rates. The main tool to influence people's expectations is communication. A number of both qualitative formal (e.g. statements and minutes) and informal (speeches) channels as well as quantitative channels, such as economic projections, are currently used by policymakers. Therefore, an in-depth understanding of the effects of central bank communication can contribute to effective monetary policy. For instance, it may help the European Central Bank to phase out the quantitative easing without causing severe instability. It is important to emphasise that this research is not about explicit forward guidance but instead focuses on the overall positive or negative sentiment (tone) about economic conditions in speeches. The paper investigates three hypotheses regarding the effect of positive or negative sentiment (tone) about economic conditions conveyed in Bank of England speeches on government bond yields in the United Kingdom: (H1) A positive sentiment (tone) about economic conditions conveyed in speeches should have a positive effect on bond yields. (H2) The impact of a speech should vary by the position of a speaker. Speeches by more senior people should have a larger effect. (H3) The stock of communication matters. If there was little communication prior to the speech, the impact of a speech should be larger.

The novelty of this paper is that it combines dictionary methods, i.e. counting occurrences of specific words, and Latent Dirichlet Allocation (LDA). First each speech is converted into a list of words that contains no punctuation or numbers since this is the only information used by LDA and dictionary methods. To find the parts of a

Gytautas Karklius

gytautas@karklius.lt

1 University of Warwick, Coventry, UK

2 University of Cambridge, Cambridge, UK 
speech talking about economic conditions, LDA is employed. LDA's main advantage is that it belongs to the group of unsupervised learning algorithms. A researcher does not specify which words define a topic. The algorithm itself creates a pre-defined number of topics, which can be then interpreted. Finally, the sentiment index is calculated by counting the number of positive and negative words in relevant parts of speeches.

After calculating the sentiment index, daily changes in the yields of two-, five- and ten-year government bonds are regressed on lagged values of the dependent variable, the sentiment index and control variables (monetary surprises and surprise components of macroeconomic data releases) in order to account for factors that can affect bond yields during the day. The estimates should be close to true values as there are not many factors that affect bond yields and are correlated with the sentiment index. Since financial variables tend to exhibit volatility clustering, generalised autoregressive conditional heteroskedasticity (GARCH) family models are used. As the sample period is long (2005-2016), an iterated cumulative sum of squares (ICSS) algorithm is employed to estimate structural breaks in variance.

The results are largely in line with Ehrmann and Fratzscher (Journal of Money, Credit and Banking, 2007), who manually code all speeches as positive, negative or neutral. The Governor and the Chief Economist's speeches have similar effects. A standard-deviation increase in the sentiment index leads to around a 1-1.7 basis point rise in the bond yields across the curve. The magnitude of the effect is similar to that for the surprise component of consumer price index (CPI) data releases. Another interesting finding is that Deputy Governors and other Monetary Policy Committee members have little or no effect on bonds, indicating that markets only react to speeches from the most senior people. Thirdly, the stock of communication matters, i.e. if there was no communication in the week prior to the speech, the effect is two- to three- times larger.

Primary limitations of this research are related to measurement error in the sentiment index. For example, 'increasing risk' and 'increasing growth' would both be classified as positive phrases even though the former is clearly negative. In addition, an absolute index, which ignores market expectations, has been used due to the lack of data. Such use is not ideal as markets should react only to the surprise component of the sentiment (the difference between the absolute index and its expectations).

There is plenty of scope to extend this research. It would be interesting to estimate the effect for a longer horizon than one day to investigate whether the effect persists. If markets need more time to absorb new information, the long-run implications may be different. Moreover, the same analysis could be repeated for more countries and types of communication. The methodology to estimate the sentiment index can be applied to other contexts.

Acknowledgments The author thanks Dr. Alexander Karalis Isaac for continued and unwavering support throughout the year, Dr. Michael McMahon for teaching computational linguistics, and Dr. Gianna Boero for general suggestions.

Open Access This article is distributed under the terms of the Creative Commons Attribution 4.0 International License (http://creativecommons.org/licenses/by/4.0/), which permits unrestricted use, distribution, and reproduction in any medium, provided you give appropriate credit to the original author(s) and the source, provide a link to the Creative Commons license, and indicate if changes were made. 